\title{
Cryptanalysis of an Enhanced Spatiotemporal Chaotic Image/Video Cryptosystem
}

\author{
Eun-Jun Yoon, ${ }^{1}$ Jeong-Woo Hong, ${ }^{2}$ Sang-Yoon Yoon, ${ }^{2}$ Dong-In Park, ${ }^{2}$ and Myung-Jin Choi ${ }^{3}$ \\ ${ }^{1}$ School of Electrical Engineering and Computer Science, Kyungpook National University, 1370 Sankyuk-Dong, \\ Buk-Gu, Daegu 702-701, Republic of Korea \\ ${ }^{2}$ Korea Institute of Science and Technology Information, 335 Gwahangno, Yuseong-Gu, Daejeon 305-806, Republic of Korea \\ ${ }^{3}$ Satellite Information Research Institute, Korea Aerospace Research Institute, 45 Eoeun-Dong, Yuseong-Gu, \\ Daejeon 305-333, Republic of Korea
}

Correspondence should be addressed to Myung-Jin Choi, prime@kari.re.kr

Received 6 September 2010; Revised 4 January 2011; Accepted 28 February 2011

Academic Editor: Ling Shao

Copyright ( 2011 Eun-Jun Yoon et al. This is an open access article distributed under the Creative Commons Attribution License, which permits unrestricted use, distribution, and reproduction in any medium, provided the original work is properly cited.

Recently, an enhanced spatiotemporal chaotic image/video cryptosystem was proposed by Rhouma and Belghith. This paper demonstrates that the enhanced cryptosystem is not secure against the following three different classical types of attacks: chosen plaintext, chosen ciphertext, and known plaintext. In the three attacks, only a pair of (plaintext/ciphertext) was needed to totally break the cryptosystem.

\section{Introduction}

Chaos theory has been applied in many different research areas, such as physics, mathematics, engineering, and biology [1]. The distinct properties of chaos, such as ergodicity, quasirandomness, sensitivity dependence on initial conditions and system parameters, have granted chaotic dynamics as a promising alternative for the conventional cryptographic algorithms. The close relationship between chaos and cryptography makes chaotic encryption a natural candidate for secure communication and cryptography [2-4]. Chaosbased cryptography is relied on the complex dynamics of nonlinear systems or maps which are deterministic but simple. Therefore, it can provide a fast and secure methods for data protection, which is crucial for multimedia data transmission over fast communication channels, such as the broadband internet communication [5-9].

The chaos-based cryptographic algorithms have suggested some new ways to develop efficient image-encryption schemes [10-14]. While most of these schemes are based on low-dimensional chaotic maps, it has been proposed recently to use high-dimensional chaos namely spatiotemporal chaos, which is modeled by one-way coupled-map lattices (OCML).
Owing to their hyperchaotic behavior, such systems are assumed to enhance the cryptosystem security [15].

A 2D CML (coupled map lattice) chaotic model [16] has a good pseudorandom property, which is regarded as of high security in data encryption and has been widely used in secure communication and multimedia encryption. For the $W \times H$ lattice, the model is defined as:

$$
\begin{gathered}
y_{n+1}^{i, j}=(1-\varepsilon) f\left(y_{n}^{i, j}\right)+\frac{1}{2} \varepsilon\left[f\left(y_{n}^{i+1, j}\right)+f\left(y_{n}^{i, j+1}\right)\right], \\
f(x)=\mu x(1-x),
\end{gathered}
$$

where $i$ and $j$ are the row and column of the lattice, respectively, $n$ is the time point, $0<\varepsilon<1, \mu=4,0 \leqslant y_{n}^{i, j} \leqslant$ $1,0 \leqslant x \leqslant 1$, and $0 \leqslant f(x) \leqslant 1$. Here, the periodic boundary conditions are $y_{n}^{i+H, j}=y_{n}^{i, j}$ and $y_{n}^{i, j+W}=y_{n}^{i, j}$.

Recently, Lian [17] proposed an efficient image/video encryption scheme based on the $2 \mathrm{D} \mathrm{CML}$ in a kind of spatiotemporal chaos system. However, Rhouma and Belghith [18] pointed out that Lian's cryptosystem is not secure to chosen ciphertext and chosen plaintext attacks $[19,20]$ because of the weaknesses in the generation of the keystream. To eliminate the weaknesses, they also proposed 
an enhanced spatiotemporal chaotic image/video cryptosystem with PCBC (propagating cipher-block chaining) mode [21-23]. Nevertheless, by analyzing the algebraic description of the enhanced cryptosystem, this study has found some drawbacks in its structure. Indeed, a chosen plaintext attack, a chosen ciphertext attack, and known plaintext attack can be done to decrypt the ciphertext without any knowledge of the main secret key values. Therefore, this paper points out three different types of attacks to break the enhanced cryptosystem described in [18]. In the proposed three attacks, only a pair of (plaintext/ciphertext) was needed to totally break the cryptosystem.

\section{Review of the Enhanced Cryptosystem}

This section gives a detailed introduction of the enhanced spatiotemporal chaotic image/video cryptosystem proposed by Rhouma and Belghith [18]. The enhanced cryptosystem can be divided into 2 phases, that is, encryption and decryption as shown in Figure 1.

2.1. Encryption Phase. A 2D CML [16] in a kind of spatiotemporal chaos is used in this phase.

(1) Given a master key $K=k_{0}, k_{1}, \ldots, k_{127}$ composed by 128 bits, the lattice is initialized as the same way in [2, Section 2.2.1].

(2) 64 chaotic sequences $\left(s_{n}^{i, j}\right)$ are generated by iterating the spatiotemporal chaos system of (1) according to the following method:

$$
s_{n}^{i, j}=\left(y_{n+t_{1}}^{i, j}+y_{n+t_{1}}^{i+1, j}\right) \bmod 1
$$

with $t_{1}>0$ to improve the initial-value sensitivity.

(3) The produced 64 sequences are quantized into one integer sequence and 63 binary sequences. The binary sequences were quantized according to the following method:

$$
s_{n}^{i, j}= \begin{cases}0 & \text { if } s_{n}^{i, j}<0.5 \\ 1 & \text { otherwise }\end{cases}
$$

for $i, j=0,1, \ldots, 7,(i, j) \neq(0,0)$, and $n=0,1, \ldots$, $N-1$, where $N$ is the converted vector length of the image of size $X \times Y$ which will be encrypted.

(4) Set $n=0$ and $s k_{n}^{i, j}=s_{n}^{i, j}$.

(5) Given the plaintext blocks $p_{n}^{i, j}$, the ciphertext blocks are generated in a similar way to the following:

$$
c_{n}^{i, j}=p_{n}^{i, j} \oplus s k_{n}^{i, j} \oplus c_{n-1}^{i, j}
$$

for $i, j=0,1, \ldots, 7$ and $n=0,1, \ldots, N-1$, where $\oplus$ is a bitwise XOR operation and $c_{-1}^{i, j}=0$.
(6) Given the generated ciphertext at index $n$, the new keystream noted $S K^{i, j}=\bigcup_{n} s k_{n}^{i, j}$ is updated as follows:

$$
s k_{n}^{i, j}=s_{n}^{i, j} \oplus p_{n-1}^{i, j}
$$

where $p_{-1}^{i, j}=0$.

(7) Set $n=n+1$ and repeat steps (5) and (6) until the encryption of all the plaintext blocks.

2.2. Decryption Phase. The decryption process is symmetric to the encryption process, but replace (4) by the following:

$$
p_{n}^{i, j}=c_{n}^{i, j} \oplus s k_{n}^{i, j} \oplus c_{n-1}^{i, j}
$$

for $i, j=0,1, \ldots, 7$ and $n=0,1, \ldots, N-1$, where $c_{-1}^{i, j}=0$.

\section{Cryptanalysis of the Enhanced Cryptosystem}

\subsection{Kerchoffs Principle and Four Classical Types of Attacks}

Kerchoffs Principle. Based on Kerchoffs principle [19, 20], there is a general assumption that the cryptanalyst who is cryptanalyzing a cryptosystem always knows exactly the design and working of the cryptosystem and then he/she can know everything about the cryptosystem except the secret key.

Four Classical Types of Attacks. There are four classical types of attacks $[18,20]$ which are ordered from the hardest types of attack to the easiest as follows.

(1) Ciphertext only attack (COA): the cryptanalyst possesses a string of ciphertext $C$.

(2) Known plaintext attack (KPA): the cryptanalyst possesses a string of plaintext $P$ and the corresponding ciphertext $C$.

(3) Chosen plaintext attack (CPA): the cryptanalyst has obtained temporary access to the encryption machinery. Hence, he/she can choose a plaintext string $P$ and construct the corresponding ciphertext string $C$.

(4) Chosen ciphertext attack (CCA): the cryptanalyst has obtained temporary access to the decryption machinery. Hence, he/she can choose a ciphertext string $C$ and construct the corresponding plaintext string $P$.

In each of these four attacks, the final objective of the cryptanalyst is to determine the key that was used. It suffices that one of the attacks is successful to consider an algorithm insecure.

3.2. PCBC (Propagating Cipher-Block Chaining) Mode. The enhanced cryptosystem is designed in the PCBC mode [21-23] of block encryption by using the previous ciphertext block $c_{n-1}^{i, j}$ and the new keystream $s k_{n}^{i, j}=s_{n}^{i, j} \oplus p_{n-1}^{i, j}$ to encrypt the present plaintext block $p_{n}^{i, j}$ at index $n$, where $s_{n}^{i, j}$ is the present key sequences and $p_{n-1}^{i, j}$ is a previous plaintext block. 


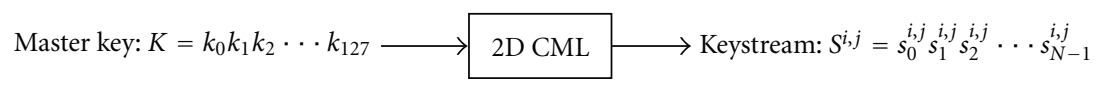

(a) Keystream generation phase

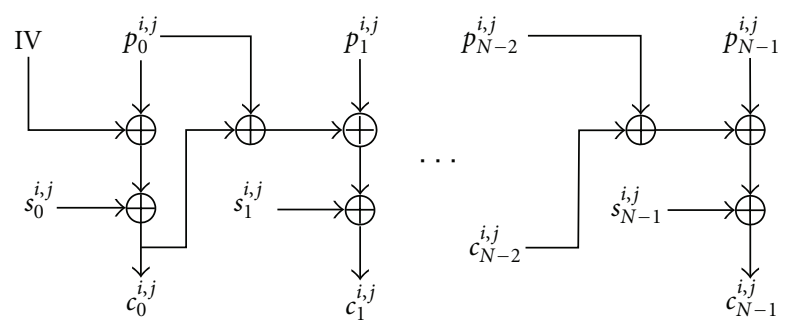

(b) Encryption phase

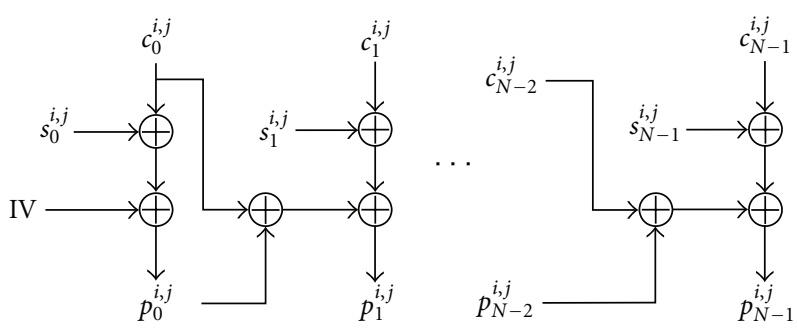

(c) Decryption phase

FIgURE 1: The enhanced spatiotemporal chaotic image/video cryptosystem.

The operation of the algorithm in the PCBC mode is described in Figure 1, and the encryption and decryption routines are as follows:

$$
\begin{array}{ll}
c_{n}^{i, j}=E_{s_{n}, j}\left(p_{n}^{i, j} \oplus p_{n-1}^{i, j} \oplus c_{n-1}^{i, j}\right), & \mathrm{IV}=p_{-1}^{i, j} \oplus c_{-1}^{i, j}, \\
p_{n}^{i, j}=D_{s_{n}^{i, j}}\left(c_{n}^{i, j}\right) \oplus p_{n-1}^{i, j} \oplus c_{n-1}^{i, j}, & \mathrm{IV}=p_{-1}^{i, j} \oplus c_{-1}^{i, j},
\end{array}
$$

where $E_{s_{i, j}}$ is the encryption block operation, $D_{s_{i j}, j}$ is the decryption block operation, and IV is the initialization vector. Here IV $=p_{-1}^{i, j} \oplus c_{-1}^{i, j}=0 \oplus 0=0$ because of $p_{-1}^{i, j}=0$ and $c_{-1}^{i, j}=0$ from steps (5) and (6) of the encryption phase.

From (7), each encryption operation based on PCBC mode can be performed as follows:

$$
\begin{aligned}
& c_{0}^{i, j}=p_{0}^{i, j} \oplus \mathrm{IV} \oplus s_{0}^{i, j}, \quad \text { IV }=p_{-1}^{i, j} \oplus c_{-1}^{i, j}=0, \\
& c_{1}^{i, j}=p_{1}^{i, j} \oplus p_{0}^{i, j} \oplus s_{1}^{i, j} \oplus c_{0}^{i, j} \\
& c_{2}^{i, j}=p_{2}^{i, j} \oplus p_{1}^{i, j} \oplus s_{2}^{i, j} \oplus c_{1}^{i, j}
\end{aligned}
$$

3.3. Chosen-Plaintext Attack (CPA). This subsection describes how the keystream $S^{i, j}=s_{0}^{i, j} s_{1}^{i, j} s_{2}^{i, j} \cdots$ of the encryption algorithm can be recovered using a chosenplaintext attack. The attack consists of two steps. First, $S^{i, j}$ is recovered. Then, the attacker uses $S^{i, j}$ to recover the plaintext $P^{i, j}$ from the arbitrary encrypted ciphertext $C^{i, j}$. The flowchart of the chosen-plaintext attack is given by Figure 2.
3.3.1. Recovering the Keystream $S^{i, j}$. Suppose that the attacker has temporary access to the encryption machinery.

(1) The attacker requests a ciphertext $D^{i, j}$ of the chosen plaintext $M^{i, j}=\bigcup_{n} m_{n}^{i, j}=00000 \cdots 000$ : a plaintext $M^{i, j}$ of the same size of the ciphertext $D^{i, j}$ constructed by the symbols $m_{n}^{i, j}=0$ for every valid $n, i$, and $j$.

(2) The attacker obtains the ciphertext $D^{i, j}=\bigcup_{n} d_{n}^{i, j}$. As can be seen that the obtained ciphertext $D^{i, j}$ is represented as follows:

$$
\begin{aligned}
& d_{0}^{i, j}=m_{0}^{i, j} \oplus \mathrm{IV} \oplus s_{0}^{i, j}, \quad \mathrm{IV}=m_{-1}^{i, j} \oplus d_{-1}^{i, j}=0, \\
& d_{1}^{i, j}=m_{1}^{i, j} \oplus m_{0}^{i, j} \oplus s_{1}^{i, j} \oplus d_{0}^{i, j}, \\
& d_{2}^{i, j}=m_{2}^{i, j} \oplus m_{1}^{i, j} \oplus s_{2}^{i, j} \oplus d_{1}^{i, j}
\end{aligned}
$$

Because $m_{n}^{i, j}=0$, the obtained ciphertext $D^{i, j}$ can be rearranged as follows:

$$
\begin{aligned}
& d_{0}^{i, j}=0 \oplus 0 \oplus s_{0}^{i, j}=s_{0}^{i, j}, \quad \text { IV }=0 \oplus 0=0, \\
& d_{1}^{i, j}=0 \oplus 0 \oplus s_{1}^{i, j} \oplus d_{0}^{i, j}=s_{1}^{i, j} \oplus d_{0}^{i, j}=s_{1}^{i, j} \oplus s_{0}^{i, j}, \\
& d_{2}^{i, j}=0 \oplus 0 \oplus s_{2}^{i, j} \oplus d_{1}^{i, j}=s_{2}^{i, j} \oplus d_{1}^{i, j}=s_{2}^{i, j} \oplus s_{1}^{i, j} \oplus s_{0}^{i, j}
\end{aligned}
$$




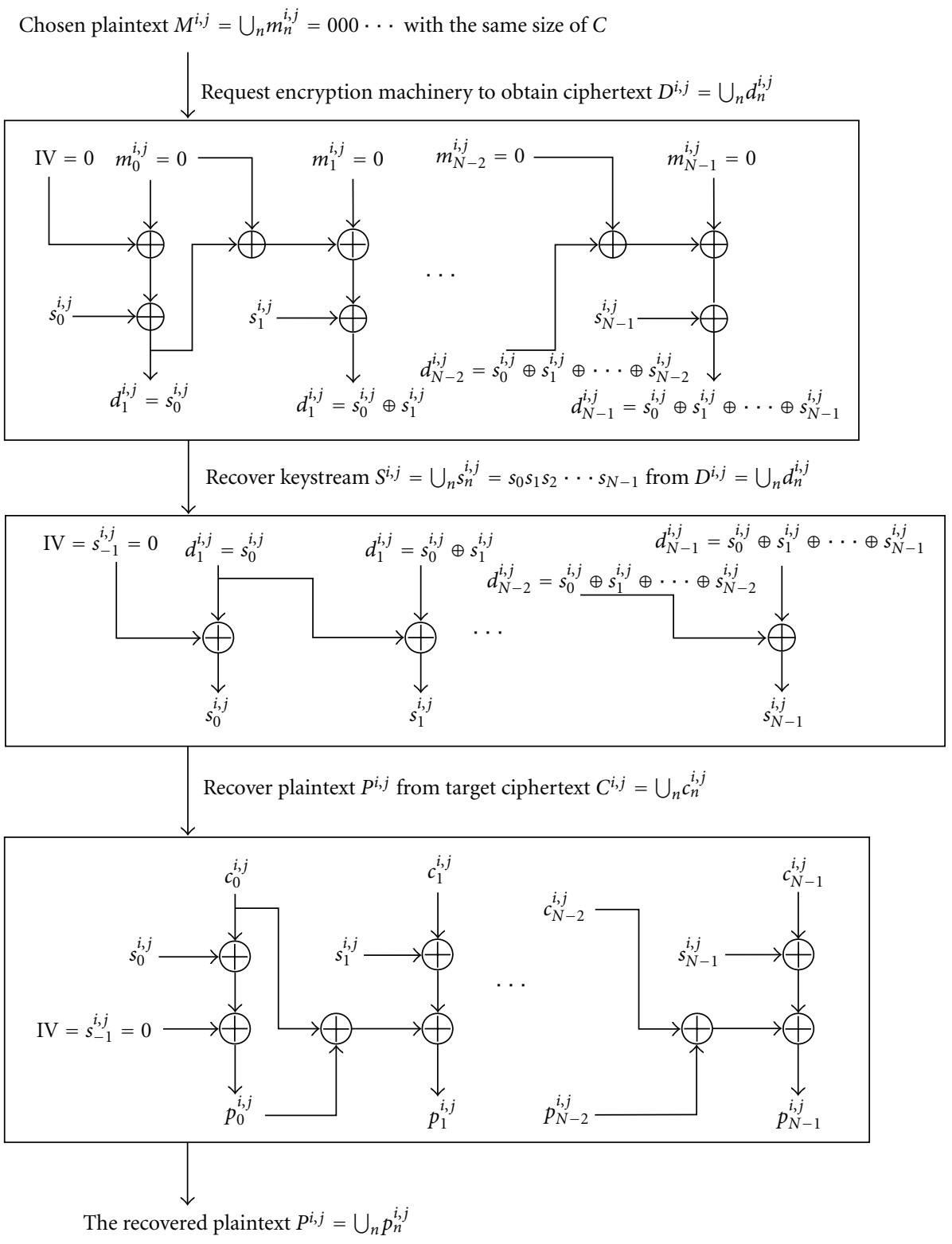

Figure 2: Flowchart of chosen-plaintext attack (CPA).

(3) The keystream $S^{i, j}=s_{0}^{i, j} s_{1}^{i, j} s_{2}^{i, j} \cdots$ can be easily recovered from $D^{i, j}$ by computing

$$
s_{n}^{i, j}=d_{n}^{i, j} \oplus d_{n-1}^{i, j},
$$

where $d_{-1}^{i, j}=0$. For example, each keystream can be obtained as follows:

$$
\begin{aligned}
& s_{0}^{i, j}=d_{0}^{i, j} \oplus d_{-1}^{i, j}=s_{0}^{i, j} \oplus 0, \\
& s_{1}^{i, j}=d_{1}^{i, j} \oplus d_{0}^{i, j}=s_{1}^{i, j} \oplus s_{0}^{i, j} \oplus s_{0}^{i, j}, \\
& s_{2}^{i, j}=d_{2}^{i, j} \oplus d_{1}^{i, j}=s_{2}^{i, j} \oplus s_{1}^{i, j} \oplus s_{0}^{i, j} \oplus s_{1}^{i, j} \oplus s_{0}^{i, j}
\end{aligned}
$$

As a result, the attacker obtains the secret keystream $S^{i, j}=$ $s_{0}^{i, j} s_{1}^{i, j} s_{2}^{i, j} \ldots$ for every $i, j=0,1, \ldots, 7,(i, j) \neq(0,0)$, and $n=$ $0,1, \ldots, N-1$.

3.3.2. Extracting the Plaintext. Once the attacker knows the secret keystream $S^{i, j}=s_{0}^{i, j} s_{1}^{i, j} s_{2}^{i, j} \cdots$ he/she can use the above decryption equation (6) to correctly reveal the plaintext $P^{i, j}=\bigcup_{n} p_{n}^{i, j}$ from an arbitrary encrypted ciphertext $C^{i, j}=$ $\cup_{n} c_{n}^{i, j}$ as

$$
p_{n}^{i, j}=c_{n}^{i, j} \oplus s k_{n}^{i, j} \oplus c_{n-1}^{i, j}
$$

for $i, j=0,1, \ldots, 7$ and $n=0,1, \ldots, N-1$, where $s k_{n}^{i, j}=$ $s_{n}^{i, j} \oplus p_{n-1}^{i, j}$ and $c_{-1}^{i, j}=0$. 


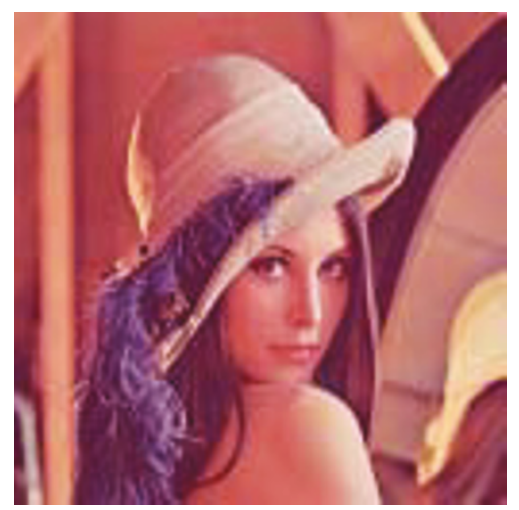

(a) Plaintext image $P$

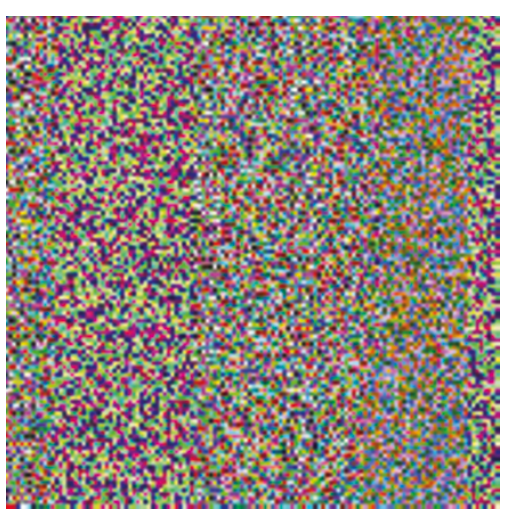

(b) Encrypted image $C$

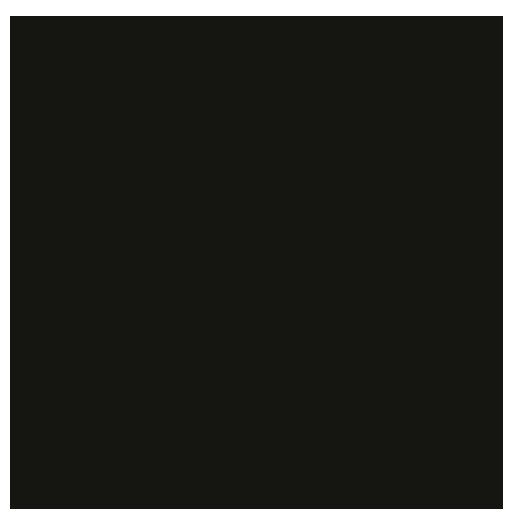

(c) Chosen plaintext image $M$

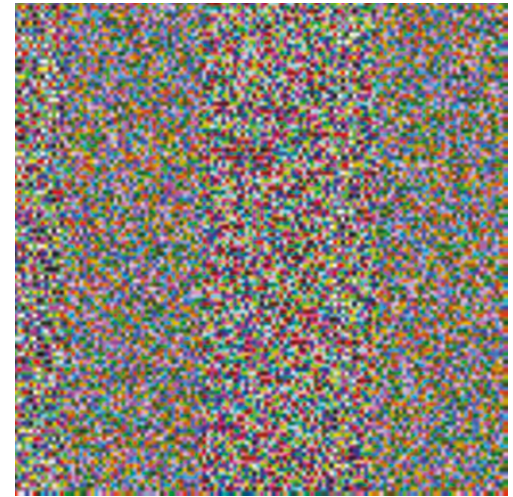

(d) Encrypted image $D$ of $M$

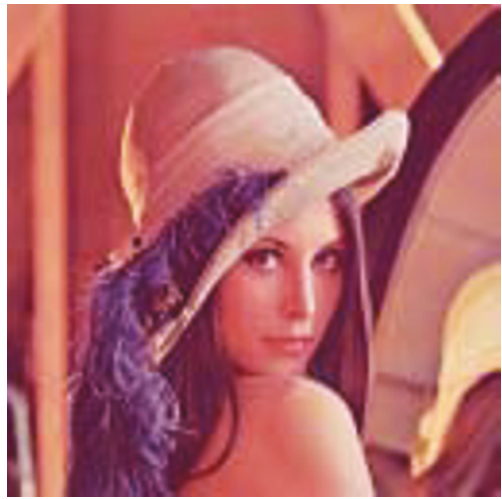

(e) Recovered image $P$ from the CPA

FIGURE 3: Simulations results of chosen-plaintext attack (CPA): (a) plaintext image $P$; (b) encrypted image $C$; (c) chosen plaintext image $M$ and (d) encrypted image $D$ of $M$ and (e) recovered image $P$ from $C$ by the CPA.

Simulations results of breaking the cryptosystem in a scenario of CPA is given in Figure 3 which shows a total recovering of the image (Lena) of size $128 \times 128$.

\subsection{Chosen-Ciphertext Attack (CCA). This subsection} describes how the keystream $S^{i, j}=s_{0}^{i, j} s_{1}^{i, j} s_{2}^{i, j} \cdots$ of the decryption algorithm can be recovered using a chosenciphertext attack. The attack consists of two steps. First, $S^{i, j}$ is recovered. Then, the attacker uses $S^{i, j}$ to recover the plaintext $P^{i, j}$ from the arbitrary encrypted ciphertext $C^{i, j}$. The flowchart of the chosen-ciphertext attack is given by Figure 4 .

3.4.1. Recovering the Keystream $S^{i, j}$. Suppose that the attacker has temporary access to the decryption machinery.

(1) The attacker requests a plaintext $M^{i, j}$ of the chosen ciphertext $D^{i, j}=\bigcup_{n} d_{n}^{i, j}=00000 \cdots 000$ : a ciphertext $D^{i, j}$ of the same size of the plaintext $M^{i, j}$ constructed by the symbols $d_{n}^{i, j}=0$ for every valid $n$, $i$ and $j$.

(2) The attacker obtains the plaintext $M^{i, j}=\bigcup_{n} m_{n}^{i, j}$. The obtained plaintext $M^{i, j}$ is represented as follows:

$$
\begin{aligned}
& m_{0}^{i, j}=d_{0}^{i, j} \oplus \mathrm{IV} \oplus s_{0}^{i, j}, \quad \mathrm{IV}=m_{-1}^{i, j} \oplus d_{-1}^{i, j}=0, \\
& m_{1}^{i, j}=d_{1}^{i, j} \oplus d_{0}^{i, j} \oplus s_{1}^{i, j} \oplus m_{0}^{i, j}, \\
& m_{2}^{i, j}=d_{2}^{i, j} \oplus d_{1}^{i, j} \oplus s_{2}^{i, j} \oplus m_{1}^{i, j}
\end{aligned}
$$

Because $d_{n}^{i, j}=0$, the obtained plaintext $M^{i, j}$ can be rearranged as follows:

$$
\begin{aligned}
& m_{0}^{i, j}=0 \oplus 0 \oplus s_{0}^{i, j}=s_{0}^{i, j}, \quad \text { IV }=0 \oplus 0=0, \\
& m_{1}^{i, j}=0 \oplus 0 \oplus s_{1}^{i, j} \oplus m_{0}^{i, j}=s_{1}^{i, j} \oplus m_{0}^{i, j}=s_{1}^{i, j} \oplus s_{0}^{i, j}, \\
& m_{2}^{i, j}=0 \oplus 0 \oplus s_{2}^{i, j} \oplus m_{1}^{i, j}=s_{2}^{i, j} \oplus m_{1}^{i, j}=s_{2}^{i, j} \oplus s_{1}^{i, j} \oplus s_{0}^{i, j}
\end{aligned}
$$

(3) The keystream $S^{i, j}=s_{0}^{i, j} s_{1}^{i, j} s_{2}^{i, j} \cdots$ can be easily recovered from $M^{i, j}$ by computing

$$
s_{n}^{i, j}=m_{n}^{i, j} \oplus m_{n-1}^{i, j},
$$




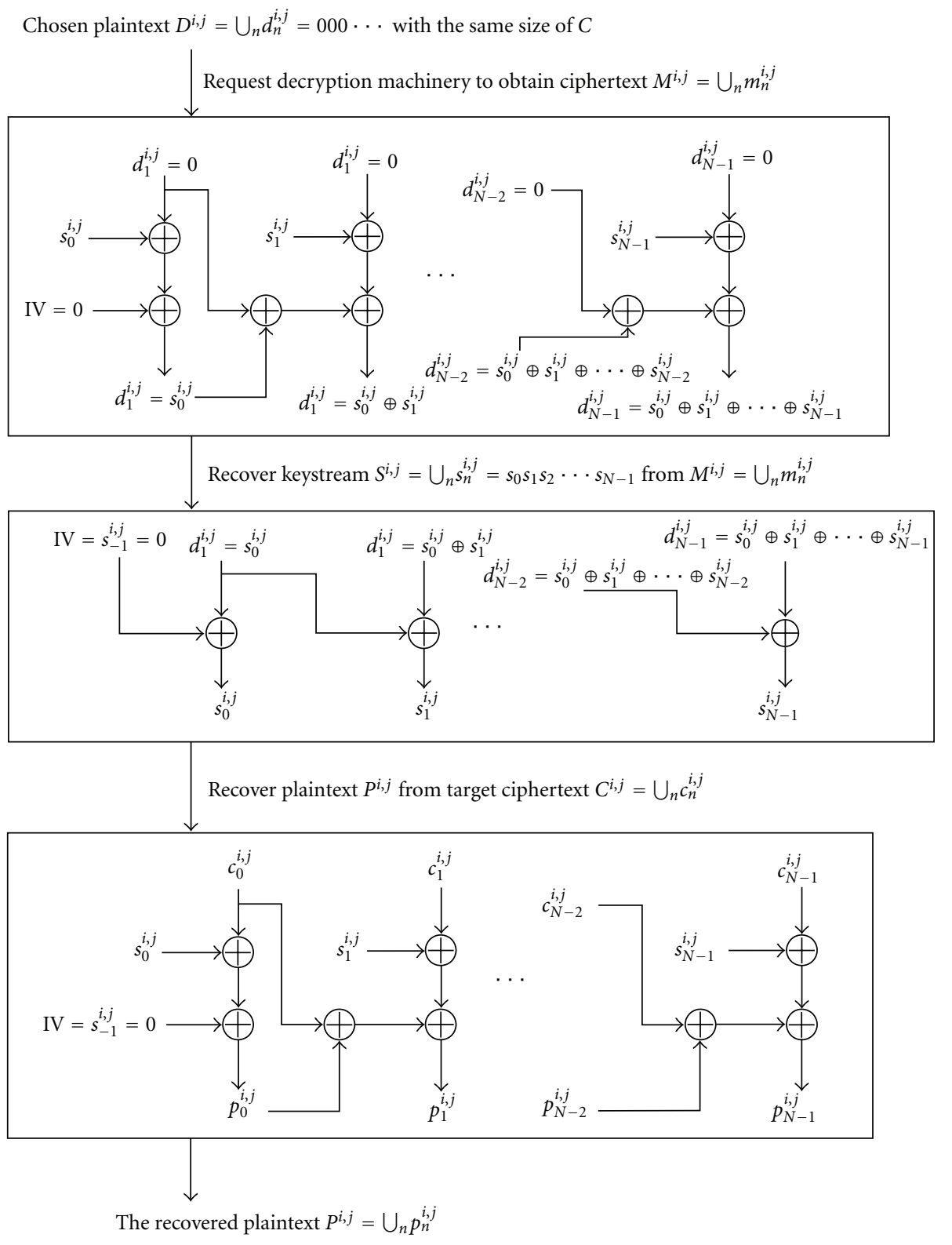

FIgURE 4: Flowchart of chosen-ciphertext attack (CCA).

where $m_{-1}^{i, j}=0$. For example, each keystream can be obtained as follows:

$$
\begin{aligned}
& s_{0}^{i, j}=m_{0}^{i, j} \oplus m_{-1}^{i, j}=s_{0}^{i, j} \oplus 0, \\
& s_{1}^{i, j}=m_{1}^{i, j} \oplus m_{0}^{i, j}=s_{1}^{i, j} \oplus s_{0}^{i, j} \oplus s_{0}^{i, j}, \\
& s_{2}^{i, j}=m_{2}^{i, j} \oplus m_{1}^{i, j}=s_{2}^{i, j} \oplus s_{1}^{i, j} \oplus s_{0}^{i, j} \oplus s_{1}^{i, j} \oplus s_{0}^{i, j}
\end{aligned}
$$

As a result, the attacker obtains the secret keystream $S^{i, j}=$ $s_{0}^{i, j} s_{1}^{i, j} s_{2}^{i, j} \ldots$ for every $i, j=0,1, \ldots, 7,(i, j) \neq(0,0)$, and $n=$ $0,1, \ldots, N-1$.
Once the attacker knows the secret keystream $S^{i, j}=$ $s_{0}^{i, j} s_{1}^{i, j} s_{2}^{i, j} \ldots$ he/she can correctly reveal the plaintext $P^{i, j}=$ $\bigcup_{n} p_{n}^{i, j}$ from an arbitrary encrypted ciphertext $C^{i, j}=\bigcup_{n} c_{n}^{i, j}$ by using the extracting plaintext phase of Section 3.3.2. Simulations results of breaking the cryptosystem in a scenario of CCA is given in Figure 5 which shows a total recovering of the image (Baboon) of size $128 \times 128$.

3.5. Known-Plaintext Attack (KPA). This subsection describes how the secret keystream $S^{i, j}=s_{0}^{i, j} s_{1}^{i, j} s_{2}^{i, j} \ldots$ of the enhanced cryptosystem can be recovered using a knownplaintext attack. In this case, the attacker dose not choose any plaintext or ciphertext. Instead, it is assumed that he/she has 


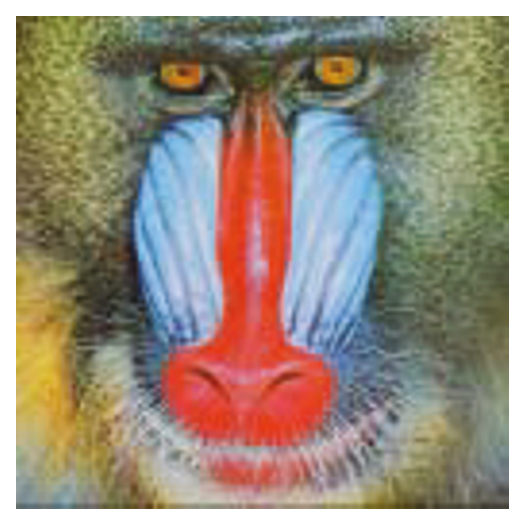

(a) Plaintext image $P$

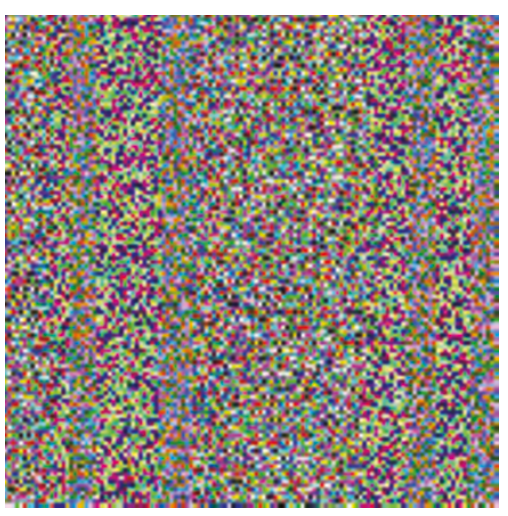

(b) Encrypted image $C$

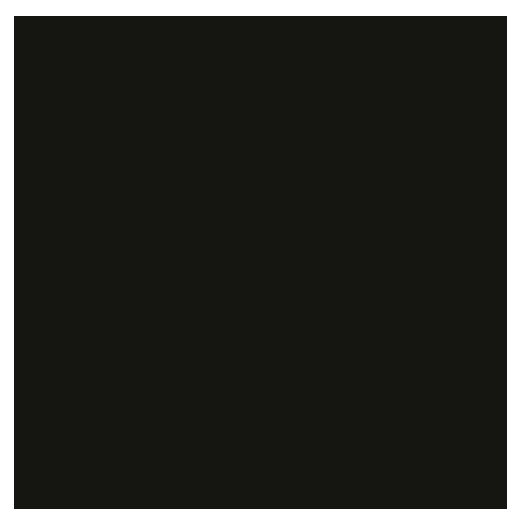

(c) Chosen ciphertext image $D$

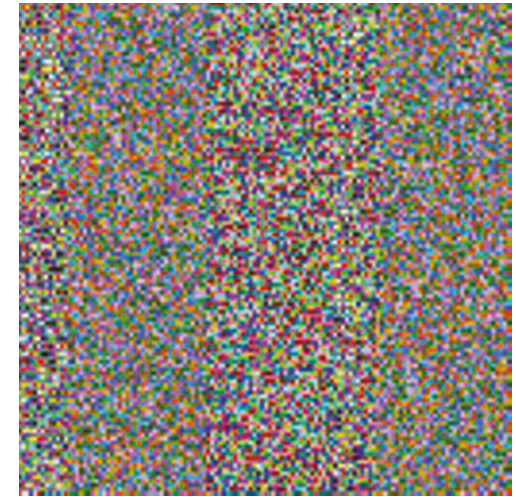

(d) Decrypted key image $M$ of $D$

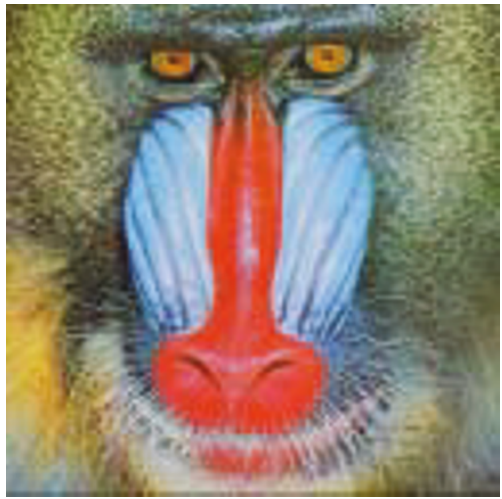

(e) Recovered image $P$ from $C$ by the CCA

Figure 5: Simulations results of chosen-ciphertext attack (CCA): (a) plaintext image $P$; (b) encrypted image $C$; (c) chosen ciphertext image $D$; (d) decrypted key image $M$ of $D$ and (e) recovered image $P$ from $C$ by the CCA.

obtained some plaintext-ciphertext pairs. The attack consists of two steps. The first step is to calculate the secret keystream key $S^{i, j}$. Then, the attacker use $S^{i, j}$ to recover the plaintext $P^{i, j}$ from the arbitrary encrypted ciphertext $C^{i, j}$. The flowchart of the known-plaintext attack is given by Figure 6 .

3.5.1. Recovering the Keystream $S^{i, j}$. Suppose that the attacker knows one plaintext-ciphertext pair $M^{i, j}=\bigcup_{n} m_{n}^{i, j}$ and $D^{i, j}=\bigcup_{n} d_{n}^{i, j}$. Then, he/she can recover the secret keystream $S^{i, j}$ by performing the following.

(1) The attacker obtains the intermediate values $s_{n}^{i, j} \oplus d_{n-1}^{i, j}$ by using (19) with the plaintext-ciphertext pair:

$$
\begin{aligned}
m_{n}^{i, j} \oplus d_{n}^{i, j} \oplus m_{n-1}^{i, j} & =m_{n}^{i, j} \oplus m_{n}^{i, j} \oplus s k_{n}^{i, j} \oplus d_{n-1}^{i, j} \oplus m_{n-1}^{i, j} \\
& =m_{n}^{i, j} \oplus m_{n}^{i, j} \oplus s_{n}^{i, j} \oplus m_{n-1}^{i, j} \oplus d_{n-1}^{i, j} \oplus m_{n-1}^{i, j} \\
& =s_{n}^{i, j} \oplus d_{n-1}^{i, j}
\end{aligned}
$$

where $d_{n}^{i, j}=m_{n}^{i, j} \oplus s k_{n}^{i, j} \oplus d_{n-1}^{i, j}$ and $s k_{n}^{i, j}=s_{n}^{i, j} \oplus m_{n-1}^{i, j}$.

(2) Let $S D^{i, j}=\bigcup_{n} s_{n}^{i, j} \oplus d_{n-1}^{i, j}$ from the result of (19), then the keystream $S^{i, j}=s_{0}^{i, j} s_{1}^{i, j} s_{2}^{i, j} \cdots$ can be easily recovered from $S D^{i, j}$ and the ciphertext $D^{i, j}=\bigcup_{n} d_{n}^{i, j}$ by computing

$$
S D^{i, j} \oplus D^{i, j}=s_{n}^{i, j} \oplus d_{n-1}^{i, j} \oplus d_{n-1}^{i, j}=s_{n}^{i, j},
$$

where $d_{-1}^{i, j}=0$.

As a result, the attacker obtains the secret keystream $S^{i, j}=$ $s_{0}^{i, j} s_{1}^{i, j} s_{2}^{i, j} \ldots$ for one plaintext-ciphertext pair, where $i, j=$ $0,1, \ldots, 7$ and $n=0,1, \ldots, N-1$.

Once the attacker knows the secret keystream $S^{i, j}=$ $s_{0}^{i, j} s_{1}^{i, j} s_{2}^{i, j} \ldots$ he/she can correctly reveal the plaintext $P^{i, j}=$ $\bigcup_{n} p_{n}^{i, j}$ from an arbitrary encrypted ciphertext $C^{i, j}=\bigcup_{n} c_{n}^{i, j}$ by using the extracting plaintext phase of Section 3.3.2. Simulation results of breaking the cryptosystem in a scenario of KPA is given in Figure 7 which shows a total recovering of the image (baboon) of size $128 \times 128$ by using the knownplain-image (jet) of the same size.

3.6. Remarks. In the enhanced spatiotemporal chaotic image/video cryptosystem, the proposed attacks are effective because $p_{-1}^{i, j} \oplus c_{-1}^{i, j}$ are both zero and so the initial vector (IV) is also zero. Although these two values are chosen as nonzero, the proposed attacks still valid because an attacker can choose nonzero same $p_{-1}^{i, j}$ and $c_{-1}^{i, j}$ which satisfy 

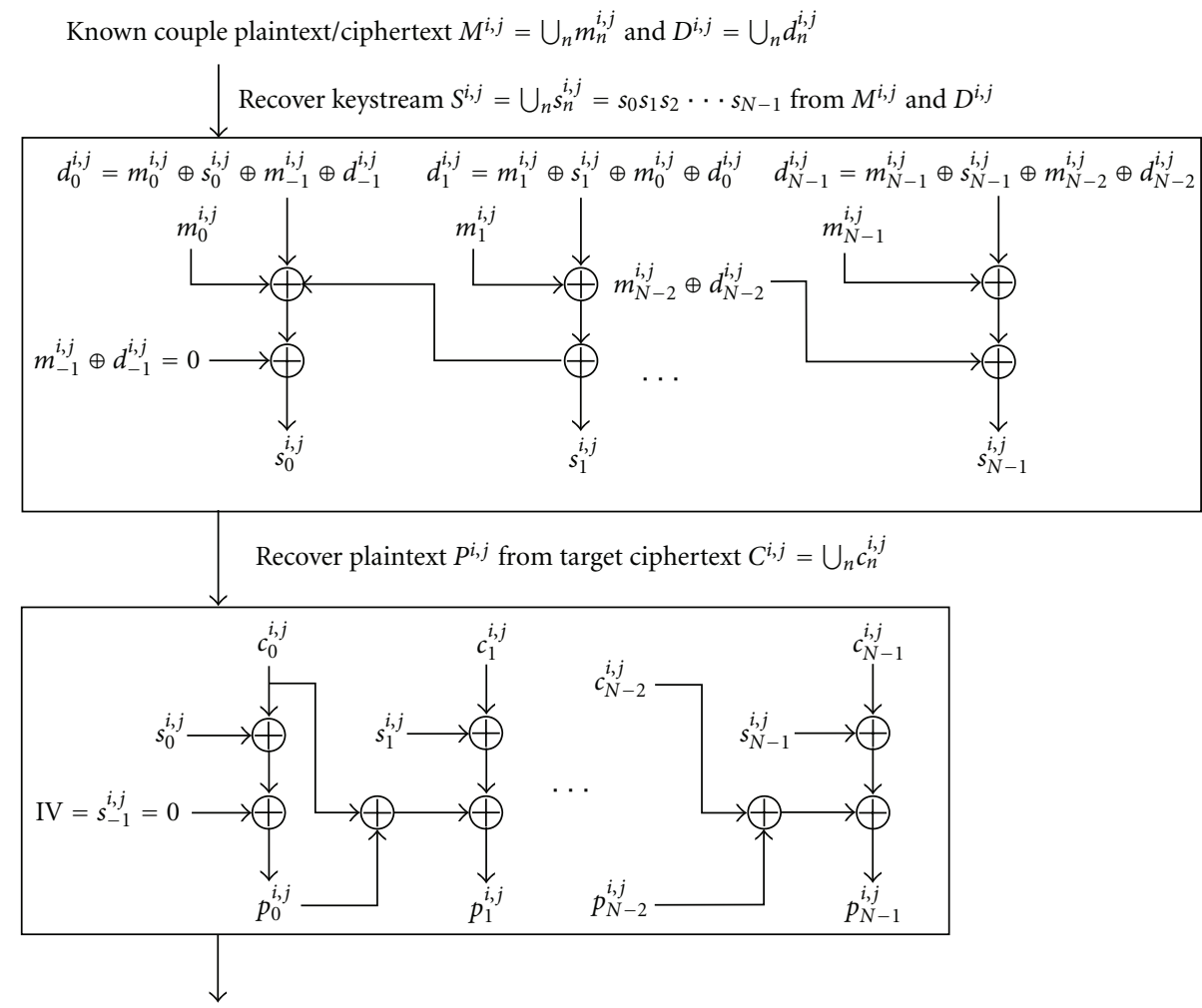

The recovered plaintext $P^{i, j}=\bigcup_{n} p_{n}^{i, j}$

FIGURE 6: Flowchart of known-plaintext attack (KPA).

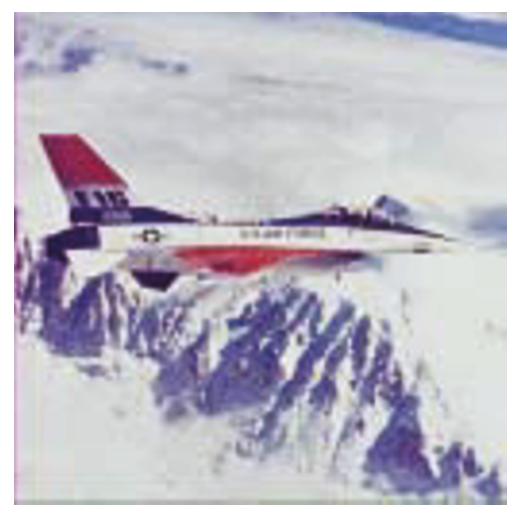

(a) Know plaintext image $M$

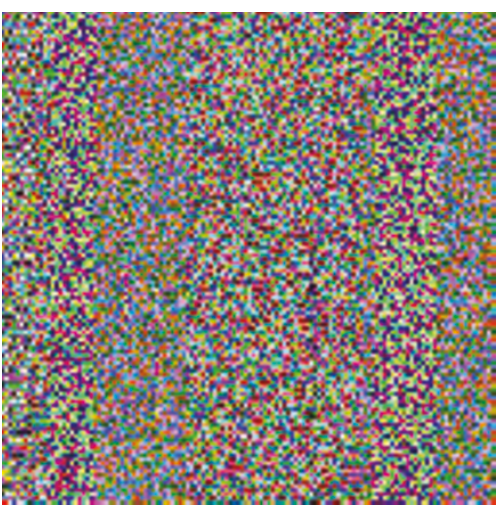

(b) Encrypted image $E$ of $M$

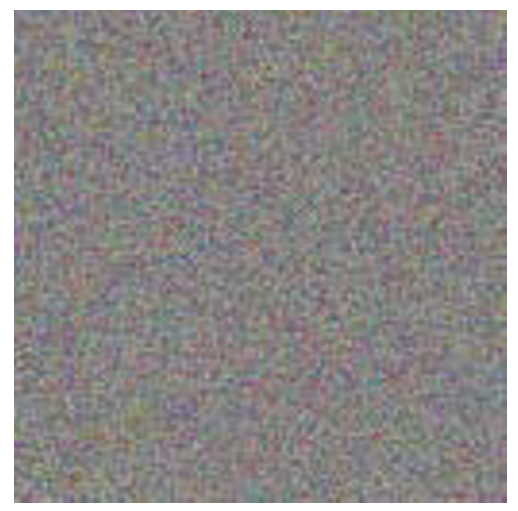

(c) Caculated key $D$ from $M$ and $E$

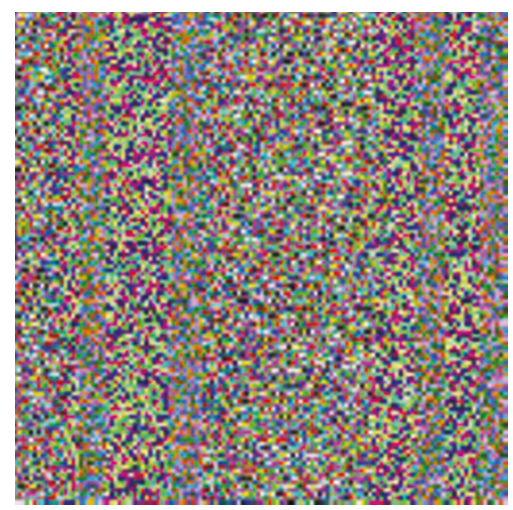

(d) Arbitrary encrypted image $C$

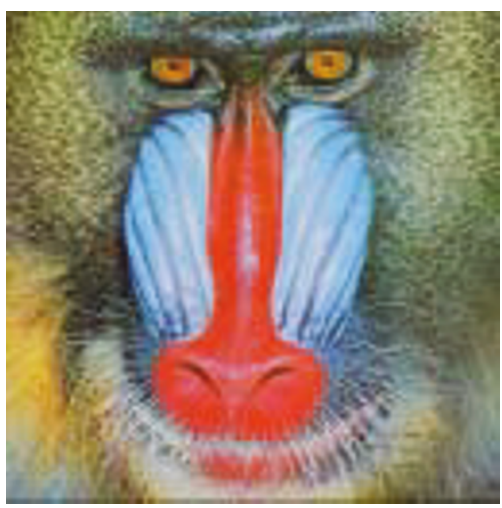

(e) Recovered image $P$ from $C$ by the KPA

FIGURE 7: Simulations results of known-plaintext attack (KPA): (a) know plaintext image $M$; (b) encrypted image $E$ of $M$; (c) calculated key $D$ from $M$ and $E$; (d) arbitrary encrypted image $C$ and (e) recovered image $P$ from $C$ by the KPA. 
$p_{-1}^{i, j} \oplus c_{-1}^{i, j}=0$. For example, let $p_{-1}^{i, j}=255(11111111)$ and $c_{-1}^{i, j}=255(11111111)$ then $p_{-1}^{i, j} \oplus c_{-1}^{i, j}=255 \oplus$ $255=11111111 \oplus 11111111=00000000=0$, let $p_{-1}^{i, j}=$ $128(10000000)$ and $c_{-1}^{i, j}=128(10000000)$ then $p_{-1}^{i, j} \oplus c_{-1}^{i, j}=$ $128 \oplus 128=10000000 \oplus 10000000=00000000=0$.

Therefore, to prevent the proposed attacks in the enhanced spatiotemporal chaotic image/video cryptosystem, IV must be known to both the sender and receiver but be unpredictable by a third party. That is, IV should be generated randomly each time and should not be reused. For maximum security, the IV should be protected against unauthorized changes. This could be done by sending the IV using ECB (electronic codebook) mode encryption with master key $K$.

\section{Conclusions}

This paper has demonstrated three separate ways to completely break an enhanced spatiotemporal chaotic image/video cryptosystem. The paper proved that the secret keystream $S^{i, j}=s_{0}^{i, j} s_{1}^{i, j} s_{2}^{i, j} \cdots$ can be easily revealed using chosen-plaintext, chosen-ciphertext, and known-plaintext attacks.

\section{Acknowledgments}

The authors would like to thank the anonymous reviewers for their helpful comments in improving the manuscript. "This research was supported by the MKE (The Ministry of Knowledge Economy), Korea, under the ITRC (Information Technology Research Center) support program supervised by the NIPA (National IT Industry Promotion Agency" (NIPA-2011-(C1090-1121-0002)).

\section{References}

[1] B. Hao, Starting with Parabolas: An Introduction to Chaotic Dynamics, Shanghai Scientific and Technological Education Publishing House, Shanghai, China, 1993.

[2] R. Brown and L. O. Chua, "Clarifying chaos: examples and counterexamples," International Journal of Bifurcation and Chaos, vol. 6, no. 2, pp. 219-249, 1996.

[3] J. Fridrich, "Symmetric ciphers based on two-dimensional chaotic maps," International Journal of Bifurcation and Chaos, vol. 8, no. 6, pp. 1259-1284, 1998.

[4] E. Solak, C. Çokal, O. T. Yildiz, and T. Biyikoğlu, "Cryptanalysis of fridrich's chaotic image encryption," International Journal of Bifurcation and Chaos, vol. 20, no. 5, pp. 1405-1413, 2010.

[5] M. Gschwandtner, A. Uhl, and P. Wild, "Transmission error and compression robustness of 2D chaotic map image encryption schemes," EURASIP Journal on Information Security, vol. 2007, Article ID 48179, 16 pages, 2007.

[6] S. Etemadi Borujeni and M. Eshghi, "Chaotic image encryption design using tompkins-paige algorithm," Mathematical Problems in Engineering, vol. 2009, Article ID 762652, 22 pages, 2009.

[7] H. Qian, J. He, Y. Zhou, and Z. Li, "Cryptanalysis and improvement of a block cipher based on multiple chaotic systems," Mathematical Problems in Engineering, vol. 2010, Article ID 590590, 14 pages, 2010.

[8] M. A. Ben Farah, A. Kachouri, and M. Samet, "Improvement of cryptosystem based on iterating chaotic map," Communications in Nonlinear Science and Numerical Simulation, vol. 16, no. 6, pp. 2543-2553, 2011.

[9] Z.-L. Zhu, W. Zhang, K.-W. Wong, and H. Yu, "A chaosbased symmetric image encryption scheme using a bit-level permutation," Information Sciences, vol. 181, no. 6, pp. 11711186, 2011.

[10] Z. H. Guan, F. Huang, and W. Guan, "Chaos-based image encryption algorithm," Physics Letters A, vol. 346, no. 1-3, pp. 153-157, 2005.

[11] S. Behnia, A. Akhshani, H. Mahmodi, and A. Akhavan, "A novel algorithm for image encryption based on mixture of chaotic maps," Chaos, Solitons \& Fractals, vol. 35, no. 2, pp. 408-419, 2008.

[12] N. K. Pareek, V. Patidar, and K. K. Sud, "Image encryption using chaotic logistic map," Image and Vision Computing, vol. 24, no. 9, pp. 926-934, 2006.

[13] C. Li, S. Li, M. Asim, J. Nunez, G. Alvarez, and G. Chen, "On the security defects of an image encryption scheme," Image and Vision Computing, vol. 27, no. 9, pp. 1371-1381, 2009.

[14] H. S. Kwok and W. K. S. Tang, "A fast image encryption system based on chaotic maps with finite precision representation," Chaos, Solitons \& Fractals, vol. 32, no. 4, pp. 1518-1529, 2007.

[15] P. Li, Z. Li, W. A. Halang, and G. Chen, "A stream cipher based on a spatiotemporal chaotic system," Chaos, Solitons \& Fractals, vol. 32, no. 5, pp. 1867-1876, 2007.

[16] R. Rhouma, S. Meherzi, and S. Belghith, "OCML-based colour image encryption,” Chaos, Solitons \& Fractals, vol. 40, no. 1, pp. 309-318, 2009.

[17] S. Lian, "Efficient image or video encryption based on spatiotemporal chaos system," Chaos, Solitons \& Fractals, vol. 40, no. 5, pp. 2509-2519, 2009.

[18] R. Rhouma and S. Belghith, "Cryptanalysis of a spatiotemporal chaotic image/video cryptosystem," Physics Letters A, vol. 372, no. 36, pp. 5790-5794, 2008.

[19] D. R. Stinson, Cryptography: Theory and Practice, CRC Press, Boca Raton, Fla, USA, 1995.

[20] G. Álvarez, F. Montoya, M. Romera, and G. Pastor, "Cryptanalysis of an ergodic chaotic cipher," Physics Letters A, vol. 311, no. 2-3, pp. 172-179, 2003.

[21] W. F. Ehrsam, C. H. W. Meyer, J. L. Smith, and W. L. Tuchman, "Message verification and transmission error detection by block chaining," US patent 4074066, 1976.

[22] J. Kohl, "The use of encryption in kerberos for network authentication," in Advances in Cryptology, vol. 435 of Lecture Notes in Computer Science, pp. 35-43, Springer, 1989.

[23] J. M. Sierra, J. C. Hernández, N. Jayaram, and A. Ribagorda, "Low computational cost integrity for block ciphers," Future Generation Computer Systems, vol. 20, no. 5, pp. 857-863, 2004. 